\title{
The Influence of Tofogliflozin on Treatment-Related Quality of Life in Patients with Type 2 Diabetes Mellitus
}

\author{
Naoto Katakami (D) - Tomoya Mita - Hidenori Yoshii - Toshihiko Shiraiwa $\cdot$ Tetsuyuki Yasuda Yosuke Okada • \\ Keiichi Torimoto • Yutaka Umayahara • Hideaki Kaneto · Takeshi Osonoi • Tsunehiko Yamamoto • \\ Nobuichi Kuribayashi · Kazuhisa Maeda · Hiroki Yokoyama • Keisuke Kosugi • Kentaro Ohtoshi · Isao Hayashi • \\ Satoru Sumitani · Mamiko Tsugawa · Kayoko Ryomoto · Hideki Taki · Tadashi Nakamura · Satoshi Kawashima • \\ Yasunori Sato $\cdot$ Hirotaka Watada · Iichiro Shimomura on behalf of the UTOPIA study investigators
}

Received: May 21, 2021 / Accepted: July 19, 2021 / Published online: August 6, 2021

(C) The Author(s) 2021

\section{ABSTRACT}

Introduction: Treatment-related quality of life (QOL) is an important aspect of diabetes management. We evaluated the influence of a sodium-glucose cotransporter 2 (SGLT2) inhibitor, tofogliflozin, on treatment-related QOL in

Details of UTOPIA study investigators are given in acknowledgement section.

Supplementary Information The online version contains supplementary material available at https:// doi.org/10.1007/s13300-021-01125-8.

N. Katakami $(\bowtie) \cdot$ I. Shimomura Department of Metabolic Medicine, Osaka University Graduate School of Medicine, 2-2, Yamadaoka, Suita, Osaka 565-0871, Japan e-mail: katakami@endmet.med.osaka-u.ac.jp

\section{N. Katakami}

Department of Metabolism and Atherosclerosis, Osaka University Graduate School of Medicine, 2-2, Yamadaoka, Suita, Osaka 565-0871, Japan

T. Mita $\cdot$ H. Watada

Department of Metabolism and Endocrinology, Juntendo University Graduate School of Medicine, Hongo 2-1-1, Bunkyo-ku, Tokyo 113-8421, Japan

H. Yoshii

Department of Medicine, Diabetology and Endocrinology, Juntendo Tokyo Koto Geriatric Medical Center, Koto-ku, Tokyo 136-0075, Japan
Japanese patients with type 2 diabetes mellitus (T2DM).

Methods: This is the prespecified subanalysis study of the "Using TOfogliflozin for Possible better Intervention against Atherosclerosis for type 2 diabetes patients (UTOPIA)" trial. Treatment-related QOL was evaluated at baseline, week 26, week 52, and week 104 after the initiation of the study using the Diabetes TherapyRelated QOL questionnaire (DTR-QOL). Among the 340 patients in the original UTOPIA study, a total of 252 patients (127, tofogliflozin group; 125 , conventional treatment group) who completed the DTR-QOL questionnaire at baseline

\section{T. Shiraiwa}

Shiraiwa Medical Clinic, 4-10-24 Hozenji,

Kashiwara, Osaka 582-0005, Japan

T. Yasuda

Department of Diabetes and Endocrinology, Osaka Police Hospital, 10-31, Kitayama-cho, Tennoji-ku, Osaka 543-0035, Japan

Y. Okada $\cdot$ K. Torimoto

First Department of Internal Medicine, School of Medicine, University of Occupational and Environmental Health, 1-1, Iseigaoka, Yahatanishiku, Kitakyushu 807-8555, Japan

Y. Umayahara

Department of Diabetes and Endocrinology, Osaka General Medical Center, 3-1-56, Bandai-Higashi, Sumiyoshi-ku, Osaka 558-8558, Japan 
were the study subjects of the current subanalysis.

Results: The tofogliflozin and conventional treatment groups exhibited almost comparable baseline clinical characteristics, while the use of antihypertensive drugs and lipid-lowering agents was significantly lower in the tofogliflozin treatment group than in the conventional treatment group. Tofogliflozin treatment increased the total score of DTR-QOL7 from baseline $(P<0.001)$, while conventional treatment did not change it. There were statistically significant differences in delta change in the total DTR-QOL7 score and DTR-QOL7 Q4, Q5, Q6, and Q7 scores from the baseline to week 104 between the treatment groups. Delta changes in HbA1c (Spearman's correlation

\section{H. Kaneto}

Department of Diabetes, Endocrinology and

Metabolism, Kawasaki Medical School, 577

Matsushima, Kurashiki, Okayama 701-0192, Japan

T. Osonoi

Nakakinen Clinic, 745-5, Nakadai, Naka, Ibaraki

311-0113, Japan

T. Yamamoto

Diabetes and Endocrinology, Kansai Rosai Hospital,

3-1-69, Inabaso, Amagasaki, Hyogo, Japan

N. Kuribayashi

Misaki Naika Clinic, 6-44-9, Futawa-higashi,

Funabashi, Chiba 274-0805, Japan

K. Maeda

Kitasenri Maeda Clinic, 4-119, Furuedai, Suita,

Osaka 565-0874, Japan

H. Yokoyama

Jiyugaoka Medical Clinic, West 6, South 6-4-3,

Obihiro, Hokkaido 080-0016, Japan

K. Kosugi

Kosugi Medical Clinic, 3-9, Tamatsukurimoto-cho,

Tennoji-ku, Osaka 543-0014, Japan

K. Ohtoshi

Otoshi Medical Clinic, 8-47, Kakudacho, Osaka Kita-

ku, Osaka 530-0017, Japan

I. Hayashi

Hayashi Clinic, 3-9-23, Koshienguchi, Nishinomiya,

Hyogo 663-8113, Japan coefficient, $\rho=-0.30, \quad P<0.001)$, fasting blood glucose $(\rho=-0.16, \quad P=0.031)$, BMI $(\rho=-0.19, P=0.008)$, and waist circumference $(\rho=-0.17, P=0.024)$ at week 104 were negatively associated with delta change in the total QOL7 score.

Conclusions: Our data indicated that tofogliflozin treatment improved treatment-related QOL compared to conventional treatment in Japanese patients with T2DM, in accordance with the improvement of major cardiovascular risk factors.Trial registration: UMIN000017607

Keywords: Quality of life (QOL); Sodiumglucose cotransporter 2 (SGLT2) inhibitor; Tofogliflozin; Type 2 diabetes mellitus

\section{S. Sumitani}

Center for Diabetes and Endocrinology, Nippon Life Hospital, 2-1-54 Enokojima, Nishi-ku, Osaka 5500006, Japan

\section{Tsugawa}

Department of Endocrinology and Metabolism, Ikeda Municipal Hospital, 3-1-18, Jonan, Ikeda, Osaka 563-8510, Japan

\section{K. Ryomoto}

Center for Diabetes Mellitus, Osaka Rosai Hospital, 1179-3 Nagasone-cho, Kita-ku, Sakai, Osaka 5918025, Japan

H. Taki

Diabetes Center, National Hospital Organization Osaka National Hospital, 2-1-14, Hoenzaka, Chuo$\mathrm{ku}$, Osaka 540-0006, Japan

\section{T. Nakamura}

Department of Internal Medicine, Kawasaki Hospital, 3-3-1, Higashiyamacho, Hyogo-ku, Kobe, Hyogo 652-0042, Japan

S. Kawashima

Kanda Naika Clinic, 5-21-3, Hannancho, Osaka Abeno-ku, Osaka 545-0021, Japan

Y. Sato

Department of Preventive Medicine and Public Health, Keio University School of Medicine, 45 Shinanomachi Shinjuku-ku, Tokyo 160-8582, Japan 


\section{Key Summary Points}

Treatment-related quality of life (QOL) is an important aspect of diabetes management.

Several studies have indicated that sodium-glucose cotransporter 2 (SGLT2) inhibitors improved treatment-related QOL in patients with type 2 diabetes.

This study aimed to evaluate the influence of an SGLT2 inhibitor, tofogliflozin, on treatment-related QOL in Japanese patients with type 2 diabetes mellitus.

Tofogliflozin treatment improved treatment-related QOL scores compared to conventional treatment in Japanese patients with type 2 diabetes mellitus, in accordance with the improvement in major cardiovascular risk factors.

Our study indicated that tofogliflozin treatment exhibited more favorable benefits than conventional treatment for QOL in Japanese patients with type 2 diabetes mellitus; this finding was fundamentally consistent with the results of previous studies, which indicated the beneficial effect of SGLT2 inhibitors on QOL.

\section{INTRODUCTION}

Treatment-related quality of life (QOL), which is closely associated with the motivation and adherence of patients [1], is an important factor in treating diabetes, since poor treatment adherence was associated with poor glycemic control and increase in risk of mortality in patients with type 2 diabetes mellitus (T2DM) [2].

Sodium-glucose cotransporter 2 (SGLT2) inhibitors, a class of antidiabetic agents, exhibit a pleiotropic effect; thus, they diminish various cardiovascular risk factors. Indeed, SGLT2 inhibitors significantly reduced major cardiovascular (CV) adverse events and/or hospitalization for heart failure in patients with T2DM [3-5]. Previous studies have also indicated that SGLT2 inhibitors are a more cost-effective option compared to other antidiabetes agents in the treatment of individuals with T2DM $[6,7]$. However, as with other classes of antidiabetic agents, this class of agents is not free from side effects, which can affect treatment-related QOL.

Several studies have evaluated effect of SGLT2 inhibitors on treatment-related QOL in patients with T2DM [8-14]. Some of them indicated that SGLT2 inhibitors such as canagliflozin [9, 12], empagliflozin [10], and dapagliflozin [11, 14] were related to the improvement of treatment-related QOL in patients with type 2 diabetes, while a few of them did not clearly show the beneficial effect of the SGLT2 inhibitor on the QOL of patients $[8,13]$.

Tofogliflozin is an SGLT2 inhibitor with a 2900-fold greater selectivity for SGLT2 than SGLT1 and the highest selectivity among all clinically developed inhibitors [15]. This potent selectivity also contributes to relatively fewer adverse events. The efficacy and safety of tofogliflozin were assessed in clinical trials: tofogliflozin significantly decreased glycated hemoglobin (HbA1c), fasting blood glucose, and body weight compared to placebo. The incidence of hypoglycemia was low, and most adverse events were classified as mild or moderate [16, 17]. Interestingly, a randomized crossover study comparing tofogliflozin and ipragliflozin used together with insulin glargine and continuous glucose monitoring revealed that tofogliflozin administration in the morning reduced the risk of nocturnal hypoglycemia because its effects almost disappeared by nighttime [18]. Moreover, although it remains unclear whether tofogliflozin reduces mortality, tofogliflozin has been shown to significantly inhibit increased brachial-ankle pulse wave velocity in patients with T2DM [19]. However, to date, no study has evaluated whether tofogliflozin induces beneficial effects on treatmentrelated QOL in patients with T2DM.

The diabetes treatment-related quality-of-life (DTR-QOL) questionnaire, a multidomain 
patient-reported outcome instrument developed in Japan, can assess the influence of diabetes treatment on patient QOL with good reliability and validity [20]. The DTR-QOL can be used regardless of the treatment method administered to patients; thus, it enables the detection of differences in patient QOL before and after a treatment switch [20].

The "Using TOfogliflozin for Possible better Intervention against Atherosclerosis for type 2 diabetes patients (UTOPIA)" was a randomized clinical trial that investigated the preventive effects of tofogliflozin on the progression of atherosclerosis in subjects with T2DM; its primary study outcome was the change in intima-media thickness (CIMT) of the common carotid artery [21, 22].

The aim of the present study is to investigate the effect of tofogliflozin on treatment-related QOL in patients with T2DM, which was a prespecified secondary outcome of the UTOPIA trial [21].

\section{METHODS}

\section{Study Design}

The original UTOPIA trial was a multicenter, prospective, randomized, open-label, blindedendpoint, multicenter, and parallel-group comparative study with a follow-up period of 104 weeks [21]. Registration of at least 340 patients was required to obtain a $90 \%$ power and detect a difference of $0.04 \mathrm{~mm}$ in CIMT between the two groups, assuming a standard deviation of $0.108,10 \%$ dropout, and a significance level of 0.05 .

The current analysis is a subanalysis from the UTOPIA to investigate the effect of tofogliflozin on treatment-related QOL in patients with T2DM. As one of the prespecified secondary outcomes, changes in the treatment-related QOL scores over the 104-week observation period were evaluated on a voluntary basis using the diabetes therapy-related QOL questionnaire. In addition, as a post hoc analysis, the association between changes in the QOL scores and changes in other clinical parameters including
HbA1c, fasting blood glucose, and body mass index (BMI) were evaluated.

This study is registered in the University Hospital Medical Information Network Clinical Trials Registry (UMIN-CTR), a nonprofit organization in Japan. It meets the requirements of the International Committee of Medical Journal Editors (UMIN000017607).

\section{Study Population}

Although the eligibility criteria of the original UTOPIA trial have been described in detail in the previous report [21] and presented as Supplementary Material in this article, the outline is as follows. The inclusion criteria were (1) Japanese patients with T2DM, inadequate glycemic control (HbA1c $\geq 6 \%$ but $<9 \%$ ), and the inability to achieve the blood glucose level stated in the Japanese Diabetes Treatment Guideline despite being on medication, other than SGLT2 inhibitors, with diet and physical therapy or only being on diet and physical therapy for at least 12 weeks; (2) no changes in antidiabetic, antithrombotic, antihypertensive medication or a therapeutic agent for dyslipidemia for at least 12 weeks before signing their consent form; (3) 30-74 years old; (4) being able to provide informed consent.

Exclusion criteria were (1) type 1 or secondary diabetes; (2) being within the perioperative period or experiencing a serious infection or injury; (3) a history of myocardial infarction, angina, stroke, or cerebral infarction; (4) severe renal dysfunction; (5) serious liver functional impairment; (6) moderate-to-severe heart failure; (7) urinary tract or genital infection; (8) pregnant, possibly pregnant, nursing, or planning to conceive a child; (9) history of hypersensitivity to the study drug; (10) present or past history of a malignant tumor (exceptions: patients who were not on medication for malignant tumor and those who exhibited no recurrence of the disease without recurrence risks during this study were allowed to participate); (11) patients prohibited from using tofogliflozin; (12) other ineligibility, as determined by an investigator. (Detailed criteria are presented in the Supplementary Material) 
A total of 340 participants with T2DM, free of apparent cardiovascular disease (CVD), were randomly and equally assigned to the tofogliflozin treatment group ( $20 \mathrm{mg}$ of tofogliflozin once daily, $n=169$ ) or the conventional treatment group that received drugs other than SGLT2 inhibitors $(n=171)$. Randomization was performed using a dynamic allocation method based on insulin use/non-use, age, and sex. In all patients, treatment was continued for achievement of the target values specified in the Treatment Guide for Diabetes edited by The Japan Diabetes Society (detailed information provided in the Supplementary Material) [23]. In the conventional treatment group, use of antidiabetic agents other than SGLT2 inhibitors was permitted: the dosage of current agents could be increased, and the addition of an alternative antidiabetic agent other than SGLT2 inhibitors was allowed 12 weeks after randomization. In the tofogliflozin group, $20 \mathrm{mg}$ of tofogliflozin once daily was initiated in addition to ongoing therapy. However, the addition of an alternative antidiabetic agent (excluding another SGLT2 inhibitor) was permitted 12 weeks after randomization. The use of antihyperlipidemic and antihypertensive drugs was allowed during the study.

Among the 340 patients in the original UTOPIA study, a total of 252 patients (127 of the tofogliflozin and 125 of the conventional treatment group, respectively) completed the DTR-QOL questionnaire at baseline, and they were the study subjects of the current subanalysis. There were significant differences in certain variables, such as total and HDL-cholesterol and use of metformin, glucagon-like peptide 1 receptor (GLP-1R) agonists, and lipid-lowering agents, between the patients who completed the DTR-QOL at baseline $(n=252)$ and those who did not $(n=88)$ (Supplementary Material Table S1).

\section{Compliance with Ethics Guidelines}

The protocol was approved by the Osaka University Clinical Research Review Committee (IRB15000038, approval number 14386, date of approval 23 April 2015) and the institutional review board of each participating institution according to the Ethical Guidelines for Medical and Health Research Involving Human Subjects issued by the Ministry of Health, Labour and Welfare in Japan. (List of IRBs is presented in the Supplementary Material.) Following enforcement of the Clinical Trials Act in April 2018, this study and its protocols were again inspected and approved by the Osaka University Clinical Research Review Committee (approval number N18007, date of approval 7 August 2019), which had obtained certification from the Minister of Health, Labour and Welfare in Japan (CRB5180007). The study was conducted in accordance with the Declaration of Helsinki, Ethical Guidelines for Medical and Health Research Involving Human Subject, the Clinical Trials Act, and other current legal regulations in Japan. Written informed consent was obtained from all participants after a full explanation of the study.

\section{Diabetes Therapy-Related QOL Questionnaire (DTR-QOL)7}

The DTR-QOL developed by Ishii is a reliable and valid questionnaire, which is a 29-item, self-administered assessment with four primary factors, presented in Japanese [20]. In the current study, we used the DTR-QOL7, a short version of the original DTR-QOL, which consisted of seven questions selected from original 29 items [24]. The items included are shown in Table 1 . The response to each question consists of a 7-point Likert-type scale that ranges from 1 (strongly agree) to 7 (strongly disagree). The scales of Q5, Q6, and Q7 were reversed so that 7 represented the highest QOL score. The DTRQOL7 was developed because of practical constraints, using data obtained from subjects with T2DM but without apparent history of CVD [24]. Although the method of selecting seven questions from the original 29 items was not based on a technical or statistical rationale, we previously confirmed that all six items other than Q2 appeared to be included in the same domain, which suggested that the structure of the DTR-QOL7 was relatively consistent [24]. 
Table 1 DTQ-QOL7 questionnaire

Q1. I am constantly concerned about time to manage my current diabetes treatment

Q2. I am bothered by weight gain with my current diabetes treatment

Q3. I am sometimes bothered by low blood glucose

Q4. I am worried about high blood glucose

Q5. Overall, I am satisfied with my current blood sugar control

Q6. With my current diabetes treatment, I am confident that I can maintain good blood glucose control

Q7. With regard to diabetes treatment, I am satisfied with current treatment methods

The total score, after simple addition of the item scores except the Q2 score, was converted to $0-100$ (best-case response $=100$; worst-case response $=0$ ). This total score had a high internal consistency based on Cronbach's alpha coefficients, and they were highly associated with the total scores of the original 29 items [24]. The Q2 score, which reflected weight gain with treatment, was separately evaluated. Each subject filled out the questionnaire and directly mailed it to the data center so that the researchers were blind to the answers. We treated the missing values according to the original DTR-QOL [20].

The DTR-QOL7 was evaluated at baseline, week 26 , week 52, and week 104 .

\section{Biochemical Tests and Safety Evaluation}

Blood samples were collected after overnight fasting. HbA1c, glucose, insulin, serum lipids, and creatinine were measured using standard techniques. Urinary albumin excretion was measured using the improved bromocresol purple method using a spot urine sample. The estimated glomerular filtration rate (eGFR) was calculated using the following formula: eGFR $\left(\mathrm{mL} / \mathrm{min}\right.$ per $\left.1.73 \mathrm{~m}^{2}\right)=194 \times$ age $-0.287 \times$ serum creatinine $-0.1094 \quad(\times 0.739$ for women) [25]. All adverse events (AEs) were recorded during the study. AEs were defined as any untoward medical occurrence in a clinical trial subject administered a medicinal product that was not necessarily related to this treatment. The details and incidence of all AEs were periodically ascertained. On the basis of the intention to treat the entire population, the safety was evaluated through recording the AEs.

\section{Statistical Analysis}

Results were presented as the mean $\pm S D$, median and interquartile range (quantile 1 and quantile 3), or number (proportion) of patients. Baseline and follow-up group comparisons were assessed with the Student's $t$ test or Wilcoxon's rank sum test for continuous variables and Fisher's exact test for categorical variables. Changes from the baseline to treatment visits were assessed using the one-sample $t$ test or Wilcoxon signed-rank test within the group. Differences in delta change in the QOL scores from baseline to weeks 52 and 104 between the groups at each visit (treatment effect) were analyzed using Wilcoxon's rank sum test. The correlation between delta changes in the total scores of DTR-QOL7, and delta changes in certain parameters from baseline to week 104 were evaluated by Spearman's rank correlation coefficient. All statistical tests were two-sided with $5 \%$ significance level. All analyses were performed using the SAS software version 9.4 (SAS Institute, Cary, NC).

\section{RESULTS}

\section{Baseline Characteristics}

The tofogliflozin $(n=127)$ and conventional treatment groups $(n=125)$ exhibited almost comparable baseline clinical characteristics, while the use of antihypertensive drugs and lipid-lowering agents were significantly lower in the tofogliflozin treatment group than in the conventional treatment group (Table 2). 
Table 2 Baseline clinical characteristics of patients in both treatment groups

\begin{tabular}{|c|c|c|c|}
\hline Parameters & Tofogliflozin group $(n=127)$ & Conventional group $(n=125)$ & $P$ value \\
\hline Sex (male) (\%) & $79(62.2)$ & $73(58.4)$ & 0.54 \\
\hline Age (years) & $61.4 \pm 9.8$ & $62.0 \pm 9.3$ & 0.63 \\
\hline Current smoking & $28(22.0)$ & $21(16.8)$ & 0.29 \\
\hline Body mass index $\left(\mathrm{kg} / \mathrm{m}^{2}\right)$ & $26.9 \pm 6.2$ & $26.8 \pm 4.6$ & 0.89 \\
\hline Waist circumference $(\mathrm{cm})$ & $93.0 \pm 13.5(n=119)$ & $93.3 \pm 11.6(n=117)$ & 0.86 \\
\hline Duration of diabetes (years) & $12.1 \pm 8.3$ & $13.0 \pm 8.8$ & 0.39 \\
\hline HbAlc (\%) & $7.4 \pm 0.7$ & $7.3 \pm 0.7$ & 0.45 \\
\hline Fasting blood glucose $(\mathrm{mmol} / \mathrm{L})$ & $7.8 \pm 1.7$ & $7.9 \pm 1.8$ & 0.55 \\
\hline C-peptide $(\mathrm{ng} / \mathrm{mL})$ & $1.91 \pm 1.24$ & $1.95 \pm 1.04$ & 0.82 \\
\hline Hypertension & $63(49.6)$ & $77(61.6)$ & 0.09 \\
\hline Systolic blood pressure $(\mathrm{mmHg})$ & $133.2 \pm 14.4(n=125)$ & $134.3 \pm 17.9(n=122)$ & 0.62 \\
\hline Diastolic blood pressure $(\mathrm{mmHg})$ & $77.3 \pm 10.5(n=125)$ & $78.8 \pm 11.2(n=122)$ & 0.28 \\
\hline Dyslipidemia & $76(59.8)$ & $85(68.0)$ & 0.18 \\
\hline Total cholesterol $(\mathrm{mmol} / \mathrm{L})$ & $5.03 \pm 0.77(n=125)$ & $4.98 \pm 0.77(n=123)$ & 0.65 \\
\hline LDL cholesterol $(\mathrm{mmol} / \mathrm{L})$ & $114.3 \pm 27.4(n=126)$ & $114.2 \pm 24.8(n=124)$ & 0.96 \\
\hline HDL cholesterol $(\mathrm{mmol} / \mathrm{L})$ & $1.41 \pm 0.37$ & $1.37 \pm 0.32$ & 0.35 \\
\hline Triglyceride $(\mathrm{mmol} / \mathrm{L})$ & $1.24(0.93,1.96)$ & $1.46[1.04,1.87]$ & 0.10 \\
\hline Diabetic retinopathy & $22(17.5)$ & $25(20.2)$ & 0.58 \\
\hline $\mathrm{eGFR}\left(\mathrm{mL} / \mathrm{min} / 1.73 \mathrm{~m}^{2}\right)$ & $81.8 \pm 22.1(n=126)$ & $82.1 \pm 25.9(n=124)$ & 0.92 \\
\hline Urinary albumin excretion $(\mathrm{mg} / \mathrm{g} / \mathrm{cre})$ & $14.9[6.3,38.7](n=122)$ & $17.7[5.3,69.4](n=123)$ & 0.74 \\
\hline \multirow[t]{2}{*}{ Diabetic nephropathy } & $38(29.9)$ & $41(32.8)$ & 0.62 \\
\hline & $(n=126)$ & $(n=124)$ & \\
\hline Use of glucose-lowering agents & $112(88.2)$ & $112(89.6)$ & 0.84 \\
\hline Use of antihypertensive drugs & $56(44.1)$ & $72(57.6)$ & 0.033 \\
\hline Use of lipid-lowering agents & $53(41.7)$ & $69(55.2)$ & 0.043 \\
\hline Use of antithrombotic agents & $14(11.0)$ & $13(10.4)$ & 1.00 \\
\hline
\end{tabular}

Data are presented as the number (\%) of patients, mean \pm standard deviation (SD) values, or median (25th and 75 th percentiles) values

$H b A 1 c$ glycated hemoglobin, SD standard deviation, LDL low-density lipoprotein, $H D L$ high-density lipoprotein 


\section{Changes in Major Clinical Parameters}

Post hoc between-group comparison of changes in clinical parameters during the treatment period were performed in 252 individuals who completed the DTR-QOL questionnaire at the baseline. Within 104 weeks, compared with the conventional group, the tofogliflozin group exhibited significantly greater reductions (value at study end - value at the baseline) in HbA1c $(-0.3 \pm 0.7 \%$ vs. $0.1 \pm 0.7 \%, P<0.001)$, fasting blood glucose $(-0.7 \pm 1.8 \mathrm{mmol} / \mathrm{L}$ vs. $0.2 \pm 1.8 \mathrm{mmol} / \mathrm{L}, \quad P<0.001), \quad$ BMI $\quad(-1.0 \pm$ $1.3 \mathrm{~kg} / \mathrm{m}^{2} \quad$ vs. $\left.-0.3 \pm 1.1 \mathrm{~kg} / \mathrm{m}^{2}, \quad P<0.001\right)$, waist circumference $(-1.0 \pm 6.2 \mathrm{~cm} \quad$ vs. $1.8 \pm 4.3 \mathrm{~cm}, P<0.001)$, systolic blood pressure $\quad(-5.1 \pm 16.1 \mathrm{mmHg} \quad$ vs. $\quad 0.7 \pm 19.2$ $\mathrm{mmHg}, P=0.014)$, and serum uric acid levels $(-0.42 \pm 0.84 \mathrm{mg} / \mathrm{dL}$ vs. $0.00 \pm 0.76 \mathrm{mg} / \mathrm{dL}$, $P<0.001)$. There was no significant difference in other clinical parameters between the groups (Supplementary Material Table S2).

During the study, concomitantly used antidiabetic agents except for biguanides and dipeptidyl peptidase 4 (DPP4) inhibitors were balanced between the conventional and tofogliflozin groups (Supplementary Material Table S3). However, antihypertensive drugs, especially angiotensin II receptor blockers, were used significantly more in the conventional group than in the tofogliflozin group during the study (Supplementary Material Table S4).

During the study, 124 patients, 57 in the tofogliflozin group and 67 in the conventional group, developed AEs $(44.9 \%$ vs. 53.6\%, $P=0.21) ; 39$ patients, 17 in the tofogliflozin group and 22 in the conventional group, developed serious AEs $(13.4 \%$ vs. $17.6 \%$, $P=0.39$ ) (Supplementary Material Table S5). In the tofogliflozin and conventional groups, 14 and 15 patients, respectively, exhibited hypoglycemic events $(11.0 \%$ vs. $12.0 \%, P=0.85)$; however, none of the affected patients experienced severe hypoglycemia.

\section{Change in DTR-QOL7 Scores}

Table 3 depicts the scores of DTR-QOL7 at each point and changes from the baseline to weeks 52 and 104. At baseline, there were no differences in the total DTR-QOL7 scores and each score of DTR-QOL7 Q1 to Q6 between the two groups.

The total score DTR-QOL7 and each score of DTR-QOL7 Q1, Q2, Q4, Q5, Q6, and Q7 were significantly increased with the tofogliflozin treatment. In contrast, the total score and score for each questionnaire except Q2 did not change with the conventional treatment. There were significant differences in delta change in the total score of DTR-QOL7 and each score of DTR-QOL7 for Q4, Q5, Q6, and Q7 from the baseline to week 104 between the treatment groups $(P<0.001,<0.005,<0.001,<0.001$, and $<0.001$, respectively).

Spearman's correlation coefficient revealed that the delta changes in HbA1c $(\rho=-0.30$, $P<0.001)$, fasting blood glucose $(\rho=-0.16$, $P=0.031)$, BMI $(\rho=-0.19, P=0.008)$, and waist circumference $(\rho=-0.17, P=0.024)$ at week 104 were negatively correlated with delta change in the total score for QOL7. Delta change in the total score for QOL7 was significantly greater in patients whose HbA1c at week 104 was less than $7 \%(n=84)$ compared to those with at least $7 \%(n=115)(8.3[0.0,19.4]$ vs. $2.8[8.3,13.9], P=0.007$ ) (Supplementary Material Table S6).

The occurrence or absence of AEs was not associated with change in the total score of QOL7 ( $\rho=-0.07, P=0.35$ ). Additionally, the occurrence of hypoglycemia was not associated with a change in total score of QOL7 ( $\rho=0.08$, $P=0.27$ ). Changes in DTR-QOL during the 104-week study period were investigated according to the treatment group among subjects without $\mathrm{AE}$ during observation period, those with AE but without SAE, and those with SAEs. In the tofogliflozin group, the total score of DTR-QOL7 was significantly increased in subjects without $\mathrm{AE}(n=55,8.3[0.0,22.2]$ (medians [range 25\%, 75\%]), $P<0.001$ ), those with AE but without SAE $(n=35,13.9$ [0.0, 22.2], $P<0.001)$, and those with SAEs $(n=12$, $18.1[0.0,25.0], P=0.016)$; and there was no significant difference in the change in DTRQOL among these three subgroups. In contrast, in the conventional treatment group, the total score of DTR-QOL7 was significantly increased 
Table 3 Effect of each treatment on DTR-QOL7 scores

\begin{tabular}{|c|c|c|c|c|}
\hline Variables & & Tofogliflozin group & Conventional group & $\begin{array}{l}P \text { value } \\
\text { (intergroup) }\end{array}$ \\
\hline \multirow{2}{*}{$\begin{array}{l}\text { Total } \\
\text { score }\end{array}$} & Baseline & $58.3[47.2,69.4](n=127)$ & $58.3[47.2,77.8](n=125)$ & 0.34 \\
\hline & Week 52 & $72.2[58.3,86.1](n=97)$ & $61.1[52.8,75.0](n=102)$ & 0.003 \\
\hline \multirow{3}{*}{$\begin{array}{c}\text { (except } \\
\text { Q2) }\end{array}$} & Week 104 & $70.8[58.3,86.1](n=102)$ & $61.1[52.8,72.2](n=98)$ & $<0.001$ \\
\hline & $\begin{array}{l}\text { Change from baseline } \\
\text { (week 52) }\end{array}$ & $11.1[2.8,22.2](n=97)^{* * *}$ & $2.8[-8.3,11.1](n=102)$ & $<0.001$ \\
\hline & $\begin{array}{l}\text { Change from baseline } \\
\text { (week 104) }\end{array}$ & $9.7[0.0,22.2](n=102)^{* * *}$ & $2.8[-8.3,8.3](n=98)$ & $<0.001$ \\
\hline \multirow[t]{5}{*}{ Q1 score } & Baseline & $5.0[4.0,7.0](n=127)$ & $5.0[4.0,6.0](n=125)$ & 0.92 \\
\hline & Week 52 & $6.0[4.0,7.0](n=97)$ & $6.0[4.0,6.0](n=101)$ & 0.015 \\
\hline & Week 104 & $6.0[4.0,7.0](n=102)$ & $5.5[4.0,7.0](n=94)$ & 0.039 \\
\hline & $\begin{array}{l}\text { Change from baseline } \\
\text { (week 52) }\end{array}$ & $0.0[0.0,2.0](n=97)^{* * *}$ & $0.0[-1.0,1.0](n=101)$ & 0.033 \\
\hline & $\begin{array}{l}\text { Change from baseline } \\
\text { (week 104) }\end{array}$ & $0.0[0.0,1.0](n=102)^{* *}$ & $0.0[-1.0,1.0](n=94)$ & 0.15 \\
\hline \multirow[t]{5}{*}{ Q2 score } & Baseline & $6.0[4.0,7.0](n=127)$ & $5.0[4.0,7.0](n=125)$ & 0.68 \\
\hline & Week 52 & $6.0[5.0,7.0](n=97)$ & $6.0[4.0,7.0](n=102)$ & 0.011 \\
\hline & Week 104 & $6.0[5.0,7.0](n=102)$ & $6.0[4.0,7.0](n=94)$ & 0.15 \\
\hline & $\begin{array}{l}\text { Change from baseline } \\
\text { (week 52) }\end{array}$ & $0.0[0.0,2.0](n=97)^{* * *}$ & $0.0[0.0,1.0](n=102)$ & 0.10 \\
\hline & $\begin{array}{l}\text { Change from baseline } \\
\text { (week 104) }\end{array}$ & $0.0[0.0,2.0](n=102)^{* *}$ & $0.0[0.0,1.0](n=94)^{*}$ & 0.36 \\
\hline \multirow[t]{5}{*}{ Q3 score } & Baseline & $7.0[6.0,7.0](n=127)$ & $7.0[6.0,7.0](n=125)$ & 0.84 \\
\hline & Week 52 & $7.0[6.0,7.0](n=97)$ & $7.0[5.0,7.0](n=102)$ & 0.89 \\
\hline & Week 104 & $7.0[6.0,7.0](n=102)$ & $7.0[6.0,7.0](n=94)$ & 0.31 \\
\hline & $\begin{array}{l}\text { Change from baseline } \\
\text { (week 52) }\end{array}$ & $0.0[0.0,1.0](n=97)$ & $0.0[0.0,1.0](n=102)$ & 0.29 \\
\hline & $\begin{array}{l}\text { Change from baseline } \\
\text { (week 104) }\end{array}$ & $0.0[-1.0,0.0](n=102)$ & $0.0[0.0,0.0](n=94)$ & 0.54 \\
\hline
\end{tabular}


Table 3 continued

\begin{tabular}{|c|c|c|c|c|}
\hline Variables & & Tofogliflozin group & Conventional group & $\begin{array}{l}P \text { value } \\
\text { (intergroup) }\end{array}$ \\
\hline \multirow[t]{5}{*}{ Q4 score } & Baseline & $4.0[2.0,5.0](n=127)$ & $4.0[3.0,5.0](n=125)$ & 0.88 \\
\hline & Week 52 & $5.0[3.0,6.0](n=97)$ & $3.0[3.0,5.0](n=102)$ & $<0.001$ \\
\hline & Week 104 & $4.5[3.0,6.0](n=102)$ & $4.0[3.0,5.0](n=98)$ & 0.010 \\
\hline & $\begin{array}{l}\text { Change from baseline } \\
\text { (week 52) }\end{array}$ & $1.0[0.0,3.0](n=97)^{* * *}$ & $0.0[-1.0,0.0](n=102)$ & $<0.001$ \\
\hline & $\begin{array}{l}\text { Change from baseline } \\
\quad \text { (week 104) }\end{array}$ & $1.0[-1.0,2.0](n=102)^{* *}$ & $0.0[-1.0,1.0](n=98)$ & 0.005 \\
\hline \multirow[t]{5}{*}{ Q5 score } & Baseline & $4.0[3.0,5.0](n=127)$ & $4.0[3.0,6.0](n=125)$ & 0.50 \\
\hline & Week 52 & $5.0[3.0,6.0](n=97)$ & $4.0[3.0,6.0](n=102)$ & 0.06 \\
\hline & Week 104 & $5.0[4.0,6.0](n=101)$ & $4.0[3.0,5.0](n=98)$ & 0.024 \\
\hline & $\begin{array}{l}\text { Change from baseline } \\
\text { (week 52) }\end{array}$ & $1.0[0.0,2.0](n=97)^{* * *}$ & $0.0[-1.0,1.0](n=102)$ & 0.002 \\
\hline & $\begin{array}{l}\text { Change from baseline } \\
\text { (week 104) }\end{array}$ & $0.0[0.0,2.0](n=101)^{* * *}$ & $0.0[-1.0,1.0](n=98)$ & 0.025 \\
\hline \multirow[t]{5}{*}{ Q6 score } & Baseline & $4.0[3.0,5.0](n=127)$ & $4.0[4.0,6.0](n=125)$ & 0.09 \\
\hline & Week 52 & $5.0[4.0,6.0](n=97)$ & $4.0[4.0,6.0](n=102)$ & 0.09 \\
\hline & Week 104 & $5.0[4.0,6.0](n=102)$ & $4.0[4.0,5.0](n=98)$ & $<0.001$ \\
\hline & $\begin{array}{l}\text { Change from baseline } \\
\text { (week 52) }\end{array}$ & $1.0[0.0,2.0](n=97)^{* * *}$ & $0.0[-1.0,1.0](n=102)$ & 0.010 \\
\hline & $\begin{array}{l}\text { Change from baseline } \\
\text { (week 104) }\end{array}$ & $1.0[0.0,2.0](n=102)^{* * *}$ & $0.0[-1.0,1.0](n=94)$ & $<0.001$ \\
\hline \multirow[t]{5}{*}{ Q7 score } & Baseline & $4.0[4.0,6.0](n=127)$ & $5.0[4.0,6.0](n=125)$ & 0.011 \\
\hline & Week 52 & $5.0[4.0,7.0](n=97)$ & $5.0[4.0,7.0](n=102)$ & 0.65 \\
\hline & Week 104 & $6.0[4.0,7.0](n=102)$ & $5.0[4.0,6.0](n=98)$ & 0.046 \\
\hline & $\begin{array}{l}\text { Change from baseline } \\
\text { (week 52) }\end{array}$ & $1.0[0.0,2.0](n=97)^{* * *}$ & $0.0[-1.0,1.0](n=102)$ & 0.001 \\
\hline & $\begin{array}{l}\text { Change from baseline } \\
\text { (week 104) }\end{array}$ & $1.0[0.0,2.0](n=102)^{* * *}$ & $0.0[1.0,1.0](n=98)$ & $<0.001$ \\
\hline
\end{tabular}

Data are expressed as the medians [range 25\%, 75\%]

Change from baseline is shown as the change in actual value between the baseline and week 104

Changes from baseline to week 104 were assessed using Wilcoxon's signed-rank test within the group. ${ }^{*} P<0.05$, ${ }^{* *} P<0.01,{ }^{* * *} P<0.001$

Differences in delta change in QOL scores from baseline to weeks 52 and 104 between the groups at each point (treatment effect) were analyzed using Wilcoxon's rank sum test 
in subjects without SAE $(n=44,5.6[2.8,11.1]$, $P=0.038$ ) but not in those with AE but without SAE $(n=39,-2.8[13.9,8.3], P=0.48)$ and in those with SAEs $(n=15,0.0 \quad[13.9,5.6]$, $P=0.75$ ); there was no significant difference in the change in the total DTR-QOL7 score among these three subgroups (Supplementary Material Table S7).

\section{DISCUSSION}

In this study, tofogliflozin treatment increased the score of DTR-QOL7 from the baseline, while conventional treatment did not change it. Indeed, there were significant differences in delta change in the score of total DTR-QOL7 and more than half of the DTR-QOL7 components from the baseline to week 104 between the treatment groups. These results indicated that tofogliflozin treatment exhibited more favorable benefits than conventional treatment for QOL in Japanese patients with T2DM.

Our finding was fundamentally consistent with previous studies, which indicated the beneficial effect of SGLT2 inhibitors on QOL [9-12, 14]. Dapagliflozin significantly improved treatment satisfaction in an open-label, singlearm observational study, which included 221 Japanese patients with T2DM [11]. In a randomized controlled trial that enrolled 253 drugnaive Japanese patients with T2DM, dapagliflozin treatment exhibited a comparable or more favorable benefit on patient QOL compared with DPP4 inhibitor treatment [14]. In addition, a pooled analysis of patient-reported outcomes data from four randomized controlled trials of canagliflozin, which primarily consisted of Caucasians, suggested that canagliflozin-treated patients generally showed improved health-related QOL [12]. Interestingly, Yoshikawa et al. reported that even intermittent empagliflozin supplementation for 24 weeks improved treatment-related QOL in 50 patients with inadequately controlled T2DM [10]. Bolge et al. evaluated the effect of canagliflozin on patient satisfaction using an internet-based questionnaire completed by healthcare providers and concluded that healthcare providers reported favorable experiences with canagliflozin and witnessed improvements in clinical outcomes and QOL of the patients [9].

However, there are also a few studies which did not clearly show the beneficial effect of the SGLT2 inhibitor on QOL [8, 13]. A 24-week, double-blind, randomized, placebo-controlled study with a 78-week extension period to evaluate the effect of dapagliflozin in combination with metformin revealed that patients maintained high QOL scores from baseline through week 102 in both the dapagliflozin + metformin group and the placebo + metformin group; there were no significant differences in the QOL scores between the two treatment groups [8]. Al-Taie et al. performed a cross-sectional study that included 170 patients with T2DM and reported that there was no statistically significant difference in the World Health Organization QOL scores between the group treated with SGLT2 inhibitors and the group that was not treated with SGLT2 inhibitors [13].

Interestingly, tofogliflozin treatment significantly improved HbA1c and fasting plasma glucose levels (Supplementary Material Table S2) and there was a significant association between the decrease in $\mathrm{HbA1c}$ and fasting plasma glucose and delta change in total DTRQOL7 scores during the treatment period. Thus, it may be reasonable to conclude that tofogliflozin treatment led to beneficial effects on patient QOL, at least partially, via improvement of glycemic control. Such an idea is consistent with previous reports that demonstrated the association of glycemic control with higher treatment-related QOL [11, 14, 20, 26, 27].

The present study showed that the Q2 score, which reflects the extent to which the patient is bothered by weight gain, was improved in the tofogliflozin group (Table 3). Indeed, tofogliflozin treatment significantly decreased body weight and waist circumference (Supplementary Material Table S2), and there was a significant association between the decrease in BMI and waist circumference and delta change in total DTR-QOL7 scores. The favorable effects of tofogliflozin on body weight may have contributed to the improvement in patient QOL, since it is generally believed that treatment- 
induced weight loss positively affects QOL [11, 14, 28-32].

The association between occurrence of AEs and change in QOL7 should be considered, since AEs negatively affect patient QOL $[33,34]$. Previous studies on tofogliflozin and other SGLT2 inhibitors revealed that SGLT2 inhibitors did not elevate the risk for hypoglycemia compared with conventional treatment $[3-5,16]$. The present study additionally showed that tofogliflozin treatment did not alter the risk of hypoglycemia compared with conventional treatment. It is reasonable that tofogliflozin treatment did not affect the score of Q3, which reflects the extent to which the patient is bothered by hypoglycemia.

A combined phase 2 and 3 randomized, placebo-controlled, double-blind, parallel-group comparative study revealed that the main AEs observed in subjects treated with tofogliflozin were hyperketonemia, ketonuria, and pollakiuria; however, most adverse events were classified as mild or moderate in severity [16]. In the present study, there was no significant difference in the occurrence of AEs between the treatment groups. Interestingly, the total score of DTR-QOL7 was significantly improved in the tofogliflozin group irrespective of the occurrence or severity of AEs. In contrast, in the conventional treatment group, the total score of DTR-QOL7 was significantly increased in patients who did not experience any AE but not in those who experienced mild AEs and SAEs. These results indicated that the QOL deterioration related to AE might have been masked by the favorable effects produced by tofogliflozin treatment.

Thus, findings from previous studies and ours suggested that improvement in DTR-QOL under tofogliflozin treatment could be dependent, at least partially, on the improvement in glycemic control and body weight loss.

The present study has certain limitations. First, this study was not a double-blind, placebo-controlled trial but rather a prospective, randomized study with open-label medications and blinded endpoint. Although the endpoint determination was blinded and conducted by expert committees, the medications were open label, which may induce unexpected bias. Furthermore, it is possible that the administration of additional antidiabetic, antilipidemic, and antihypertensive agents, which was more frequent in the control group at baseline and during the treatment periods (Supplementary Material Tables S3 and S4), may have affected the outcomes. Second, the study was a subanalysis, where a relatively small number of subjects were included from the original study, because the questionnaire was completed on a voluntary basis. There were significant differences in certain variables at baseline between those who underwent DTR-QOL questionnaire and those who did not (Supplementary Material Table S1), which was not irrelevant to bias. Therefore, results should be interpreted with caution and further investigation in a largescale study that uses changes in treatment-related QOL over time as the primary outcome is required. This may cause a selection bias. Third, we evaluated treatment-related QOL only by DTR-QOL7. The small number of questions is a weakness in terms of evaluating a wide range of influences of diabetes treatment on QOL. Fourth, in this study, the association between QOL and the occurrence of AEs as two categories (e.g., $\mathrm{AE}(+)$ or $\mathrm{AE}(-)$ during the 104-week study period) was evaluated; however, the time to onset of AEs was not considered. Therefore, the correlation between AEs and QOL might be overestimated or underestimated. Finally, multiple statistical analyses were performed on these subjects, which would generate false positive results derived from multiple testing. Further studies are required to confirm our findings.

\section{CONCLUSIONS}

Our data indicated that tofogliflozin treatment improved treatment-related QOL compared to conventional treatment in Japanese patients with type 2 diabetes, in association with body weight loss and improvement of glycemic control. 


\section{ACKNOWLEDGEMENTS}

The authors thank all the staff and patients who participated in this study. The authors gratefully acknowledge the assistance of D. Takayama and H. Yamada (Soiken Holdings Inc., Tokyo, Japan).

Funding. Financial support for this study, including the fees for the journal's Rapid Service Fee, was provided by Kowa Co. Ltd., Tokyo, Japan.

Editorial Assistance. The authors gratefully acknowledge the assistance of Editage (www.editage.com) for English language editing (funded by Kowa Co. Ltd., Tokyo, Japan).

Authorship. All named authors meet the International Committee of Medical Journal Editors (ICMJE) criteria for authorship for this article, take responsibility for the integrity of the work as a whole, and have given their approval for this version to be published.

Author Contribution. All authors contributed to the study design and were involved at all stages of manuscript development. NK drafted the manuscript. YS contributed to the analysis of research data. All authors were involved in analysis and interpretation of data, reviewed/edited the manuscript and approved the final manuscript. IS and HW were the principal guarantors of this work; they have full access to all the data, and they take responsibility for the integrity of the data and accuracy of data analysis.

List of Investigators. Hayashi Clinic: I. Hayashi; Ikeda Municipal Hospital: M. Tsugawa; Jiyugaoka Medical Clinic: H. Yokoyama; Juntendo Tokyo Koto Geriatric Medical Center: H. Yoshii; Juntendo University Graduate School of Medicine: K. Komiyama, T. Mita, T. Shimizu; Kansai Rosai Hospital: T. Yamamoto; Kanda Naika Clinic: S. Kawashima; Kawasaki Hospital: T. Nakamura; Kawasaki Medical School: S. Kamei, T. Kinoshita, M. Shimoda; Kitasenri Maeda Clinic: K. Maeda; Kosugi Medical Clinic: K. Kosugi; Misaki Naika Clinic: H. Yoshii;
NakaKinen Clinic: H. Ishida, T. Osonoi, M. Saito, A. Tamazawa; Nissay Hospital: S. Sumitani; Osaka General Medical Center: N. Fujiki, Y. Fujita, S. Shimizu, Y. Umayahara; National Hospital Organization Osaka National Hospital: K. Kato; Osaka Police Hospital: Y. Irie, R. Kataoka, T. Yasuda; Osaka Rosai Hospital: Y. Kiyohara, M. Ohashi, K. Ryomoto, Y. Takahi; Osaka University Graduate School of Medicine: Y. Fujishima, Y. Fujita, A. Fukuhara, K. Fukui, Y. Hosokawa, A. Imagawa, H. Iwahashi, K. Mukai, N. Katakami, T. Katsura, D. Kawamori, T. Kimura, S. Kobayashi, J. Kozawa, F. Kubo, N. Maeda, T. Matsuoka, K. Miyashita, S. Nakata, H. Ninomiya, H. Nishizawa, Y. Okuno, M. Otsuki, F. Sakamoto, S. Sasaki, I. Sato, N. Shimo, I. Shimomura, M. Takahara, T. Takano, A. Tokunaga, S. Uno, M. Yamaoka, S. Yoneda; Otoshi Medical Clinic: K. Ohtoshi; Shiraiwa Medical Clinic: T. Shiraiwa; University of Occupational and Environmental Health, Japan: M. Hajime, K. Koikawa, F. Kuno, A. Kurozumi, K. Matsushita, M. Narisawa, K. Tanaka, K. Sugai, Y. Okada, K. Torimot

Disclosures. Naoto Katakami was a staff member of the endowed chair established by funds from Kowa Co. Ltd. and has received research funds from MSD and lecture fees from Astellas Pharma Inc., AstraZeneca K.K., Boehringer Ingelheim, Daiichi Sankyo Inc., Eli Lilly, Kowa Pharmaceutical Co., Kyowa Hakko Kirin Co. Ltd., Mitsubishi Tanabe Pharma Co., Novartis Pharmaceuticals, Novo Nordisk Pharma, Ono Pharmaceutical Co., Taisho Toyama Pharmaceutical Co., Takeda Pharmaceutical Co., Sanofi-Aventis, and Shionogi \& Co. Tomoya Mita has received lecture fees from Astellas Pharma Inc., Daiichi Sankyo Inc., Eli Lilly, Kowa Pharmaceutical Co., Kyowa Hakko Kirin Co. Ltd., Mitsubishi Tanabe Pharma Co., Novo Nordisk Pharma, Ono Pharmaceutical Co. Ltd., Takeda Pharmaceutical Company Ltd., and Sanofi-Aventis; scholarship donations from MSD K.K., Astellas Pharma Inc., AstraZeneca K.K., Ono Pharmaceutical Co. Ltd., Kyowa Hakko Kirin Co. Ltd., Sanofi-Aventis K.K., Daiichi Sankyo Company, Limited, Sumitomo Dainippon Pharma Co., Ltd., Takeda Pharmaceutical Company Limited, Mitsubishi Tanabe 
Pharma Corporation, Terumo Corporation, Nippon Boehringer Ingelheim Co. Ltd., Novo Nordisk Pharma Ltd., Pfizer Japan Inc., Benefit One Health Care Inc., Mochida Pharmaceutical Co. Ltd., and Nitto Boseki Co. Ltd.; and endowed chair funding from MSD K.K. and Takeda Pharmaceutical Company Limited. Toshihiko Shiraiwa has received lecture fees from Sanofi-Aventis K.K. and Takeda Pharmaceutical Company Limited and research funding from Novo Nordisk Pharma Ltd., SanofiAventis K.K., Takeda Pharmaceutical Company Limited, AstraZeneca K.K., Nippon Boehringer Ingelheim Co. Ltd., and Mitsubishi Tanabe Pharma Corporation. Tetsuyuki Yasuda received lecture fees from Nippon Boehringer Ingelheim Co. Ltd. and Sanofi-Aventis K.K. Yosuke Okada has received lecture fees from Astellas Pharma Inc., MSD K.K., Ono Pharmaceutical Co. Ltd., Mitsubishi Tanabe Pharma Corporation, Bayer Holding Ltd., Novartis Pharmaceuticals Corp., Novo Nordisk Pharma Ltd., Eli Lilly Japan K.K., and Kissei Pharmaceutical Co. Ltd. and research funding from Kowa Pharmaceutical Co. Ltd. and Mitsubishi Tanabe Pharma Corporation. Hideaki Kaneto has received lecture fees from Nippon Boehringer Ingelheim Co. Ltd., SanofiAventis K.K., Novo Nordisk Pharma Ltd., Eli Lilly Japan K.K., MSD K.K., Ono Pharmaceutical Co. Ltd., Takeda Pharmaceutical Company Limited, Daiichi Sankyo Company, Limited, Mitsubishi Tanabe Pharma Corporation, AstraZeneca K.K., Astellas Pharma Inc., Novartis Pharmaceuticals Corp., and Sumitomo Dainippon Pharma Co.; scholarship donations from Novo Nordisk Pharma Ltd., Nippon Boehringer Ingelheim Co., Ltd., Sumitomo Dainippon Pharma Co. Ltd., Ono Pharmaceutical Co. Ltd., Sanofi-Aventis K.K., Eli Lilly Japan K.K., Astellas Pharma Inc., Daiichi Sankyo Company, Limited, Mitsubishi Tanabe Pharma Corporation, MSD K.K., Takeda Pharmaceutical Company Limited, AstraZeneca K.K., Mochida Pharmaceutical Co. Ltd., Taisho Toyama Pharmaceutical Co. Kissei Pharmaceutical Co. Ltd., and Kyowa Hakko Kirin Co. Ltd; and research funding from Taisho Pharmaceutical Co., Sumitomo Dainippon Pharma Co., and Nippon Boehringer Ingelheim Co. Takeshi Osonoi has received lecture fees from Takeda
Pharmaceutical Company Limited, Astellas Pharma Inc., Novo Nordisk Pharma Ltd., Sanwa Kagaku Kenkyusho Co. Ltd.; manuscript fees from Sanwa Kagaku Kenkyusho Co. Ltd.; and research funding from Takeda Pharmaceutical Company Limited, Novo Nordisk Pharma Ltd., Astellas Pharma Inc., Sanwa Kagaku Kenkyusho Co. Ltd., Mitsubishi Tanabe Pharma Corporation, Nippon Boehringer Ingelheim Co. Ltd., Taisho Pharmaceutical Co. Ltd., Eli Lilly Japan K.K., Daiichi Sankyo Company Limited, Bayer Holding Ltd., Kowa Pharmaceutical Co. Ltd., and AbbVie Inc. Nobuichi Kuribayashi has received lecture fees from Takeda Pharmaceutical Company Limited, Sanofi-Aventis K.K., Novo Nordisk Pharma Ltd., MSD K.K., and Mitsubishi Tanabe Pharma Corporation. Satoru Sumitani has received lecture fees from Sumitomo Dainippon Pharma Co. Ltd. Yasunori Sato has received lecture fees from Mochida Pharmaceutical Co. Ltd. Hirotaka Watada has received lecture fees from Sumitomo Dainippon Pharma Co. Ltd., Bayer Yakuhin Ltd. SanofiAventis K.K., MSD K.K., Astellas Pharma Inc., Takeda Pharmaceutical Company Ltd., Mitsubishi Tanabe Pharma Co., AstraZeneca K.K., Nippon Boehringer Ingelheim Co. Ltd., Eli Lilly Japan K.K., Novo Nordisk Pharma Ltd., Sanwa Kagaku Kenkyusho Co. Ltd., Kowa Co. Ltd., Novartis Pharmaceuticals Corp., Daiichi Sankyo Company Ltd, Kyowa Hakko Kirin Co. Ltd., Ono Pharmaceutical Co. Ltd., and Kissei Pharmaceutical Co. Ltd.; and research support from Novartis Pharmaceuticals Corp., Otsuka Pharmaceutical Co. Ltd., Eli Lilly Japan K.K., MSD K.K., Astellas Pharma Inc., Bayer Yakuhin Ltd. Teijin Pharma Ltd., Ono Pharmaceutical Co. Ltd., Kissei Pharmaceutical Co. Ltd., Kyowa Hakko Kirin Co. Ltd., Kowa Pharmaceutical Co. Ltd., Sanofi-Aventis K.K., Sanwa Kagaku Kenkyusho Co. Ltd., Daiichi Sankyo Company Ltd., Sumitomo Dainippon Pharma Co. Ltd., Taisho Toyama Pharmaceutical Co. Ltd., Takeda Pharmaceutical Company Ltd., Mitsubishi Tanabe Pharma Corporation, Nippon Boehringer Ingelheim Co. Ltd., Novo Nordisk Pharma Ltd., Pfizer Japan Inc., Shionogi \& Co. Ltd., Yakult, and Kissei Pharmaceutical Co., Ltd. Iichiro Shimomura has received lecture fees from Astellas Pharma Inc., AstraZeneca K.K., MSD K.K., Ono 
Pharmaceutical Co., Kyowa Kirin Co. Ltd., Kowa Company Ltd., Sanofi K.K., Sanwa Kagaku Kenkyusho Co., Daiichi Sankyo Co., Takeda Pharma K.K., Mitsubishi Tanabe Pharma Co., Teijin Pharma, Eli Lilly Japan K.K., Nippon Boehringer Ingelheim Co., Novartis Pharma K.K., Novo Nordisk Pharma, Mochida Pharmaceutical Co., Taisho Pharmaceutical Co. Ltd., Nippon Chemiphar Co. Ltd., Covidien Japan Inc., Amgen Astellas Biopharma K.K., KOBAYASHI Pharmaceutical Co. Ltd., Dainippon Sumitomo Pharma Co., Rohto Pharmaceutical Co. Ltd.; research funds from Astellas Pharma Inc., MSD K.K, Ono Pharmaceutical Co., Kaken Pharmaceutical Co., Kyowa Kirin Co. Ltd., Sanofi K.K., Shionogi \& Co., Daiichi Sankyo Co., Dainippon Sumitomo Pharma Co., Takeda Pharma K.K., Mitsubishi Tanabe Pharma Co., Teijin Pharma, Novartis Pharma K.K., Novo Nordisk Pharma, Eli Lilly Japan K. K, Kowa Company Ltd.; and consulting fees from AstraZeneca K.K., MSD K.K., Taisho Pharmaceutical Co. Ltd., Novo Nordisk Pharma, and Lotte Co. Ltd. Hidenori Yoshii, Keiichi Torimoto, Yutaka Umayahara, Tsunehiko Yamamoto, Kazuhisa Maeda, Hiroki Yokoyama, Keisuke Kosugi, Kentaro Ohtoshi, Isao Hayashi, Mamiko Tsugawa, Kayoko Ryomoto, Hideki Taki, Tadashi Nakamura, and Satoshi Kawashima declare that they have no conflicts of interest.

Compliance with Ethics Guidelines. The protocol was approved by the Osaka University Clinical Research Review Committee (IRB15000038, approval number 14386, date of approval 23 April 2015) and the institutional review board of each participating institution according to the Ethical Guidelines for Medical and Health Research Involving Human Subjects issued by the Ministry of Health, Labour and Welfare in Japan. (List of IRBs is presented in the Supplementary Material of this article.) Following enforcement of the Clinical Trials Act in April 2018, this study and its protocols were again inspected and approved by the Osaka University Clinical Research Review Committee (approval number N18007, date of approval 7 August 2019), which had obtained certification from the Minister of Health, Labour and Welfare in Japan (CRB5180007). The study was conducted in accordance with the Declaration of Helsinki, Ethical Guidelines for Medical and Health Research Involving Human Subject, the Clinical Trials Act, and other current legal regulations in Japan. Written informed consent was obtained from all participants after a full explanation of the study.

Data Availability. The datasets generated during and/or analyzed during the current study are available from the corresponding author on reasonable request.

Open Access. This article is licensed under a Creative Commons Attribution-NonCommercial 4.0 International License, which permits any non-commercial use, sharing, adaptation, distribution and reproduction in any medium or format, as long as you give appropriate credit to the original author(s) and the source, provide a link to the Creative Commons licence, and indicate if changes were made. The images or other third party material in this article are included in the article's Creative Commons licence, unless indicated otherwise in a credit line to the material. If material is not included in the article's Creative Commons licence and your intended use is not permitted by statutory regulation or exceeds the permitted use, you will need to obtain permission directly from the copyright holder. To view a copy of this licence, visit http://creativecommons.org/licenses/by$\mathrm{nc} / 4.0 /$.

\section{REFERENCES}

1. Ishii $\mathrm{H}$, Anderson JH Jr, Yamamura A, Takeuchi M, Ikeda I. Improvement of glycemic control and quality-of-life by insulin lispro therapy: assessing benefits by ITR-QOL questionnaires. Diabetes Res Clin Pract. 2008;81:169-78.

2. Ho PM, Rumsfeld JS, Masoudi FA, et al. Effect of medication nonadherence on hospitalization and mortality among patients with diabetes mellitus. Arch Intern Med. 2006;166:1836-41.

3. Zinman B, Wanner C, Lachin JM, et al. Empagliflozin, cardiovascular outcomes, and mortality in type 2 diabetes. N Engl J Med. 2015;373:211721-8. 
4. Neal B, Perkovic V, Mahaffey KW, et al. Canagliflozin and cardiovascular and renal events in type 2 diabetes. N Engl J Med. 2017;377:644-57.

5. Wiviott SD, Raz I, Bonaca MP, et al; DECLARE-TIMI 58 Investigators. Dapagliflozin and cardiovascular outcomes in type 2 diabetes. $\mathrm{N}$ Engl J Med. 2019;380:347-57.

6. Rahmana W, Solinskyb PJ, Munirc KM, Lamosc EM. Pharmacoeconomic evaluation of sodium-glucose transporter-2 (SGLT2) inhibitors for the treatment of type 2 diabetes. Expert Opin Pharmacother. 2019;20(2):151-61.

7. Ehlers LH, Lamotte M, Monteiro S, et al. The costeffectiveness of empagliflozin versus liraglutide treatment in people with type 2 diabetes and established cardiovascular disease. Diabetes Ther. 2021;12(5):1523-34.

8. Grandy S, Langkilde AM, Sugg JE, Parikh S, Sjöström CD. Health-related quality of life (EQ-5D) among type 2 diabetes mellitus patients treated with dapagliflozin over 2 years. Int J Clin Pract. 2014;68: 486-94. https://doi.org/10.1111/ijcp.12341.

9. Bolge SC, Flores NM, Huang S, Cai J. Health care provider experience with canagliflozin in real-world clinical practice: favorability, treatment patterns, and patient outcomes. Int J Gen Med. 2017;10: 177-87. https://doi.org/10.2147/IJGM.S138583.

10. Yoshikawa F, Kumashiro N, Shigiyama F, et al. Efficacy of intermittent empagliflozin supplementation on dietary self-management and glycaemic control in patients with poorly controlled type 2 diabetes: a 24-week randomized controlled trial. Diabetes Obes Metab. 2019;21:303-11.

11. Nakajima H, Okada S, Mohri T, et al. Dapagliflozin improves treatment satisfaction in overweight patients with type 2 diabetes mellitus: a patient reported outcome study (PRO study). Diabetol Metab Syndr. 2018;10:11. https://doi.org/10.1186/ s13098-018-0313-X.

12. Cai J, Delahanty LM, Akapame S, Slee A, Traina S. Impact of canagliflozin treatment on health-related quality of life among people with type 2 diabetes mellitus: a pooled analysis of patient-reported outcomes from randomized controlled trials. Patient. 2018;11:341-52. https://doi.org/10.1007/s40271017-0290-4.

13. Al-Taie N, Maftei D, Kautzky-Willer A, Krebs M, Stingl H. Assessing the health-related quality of life in type 2 diabetes patients treated with insulin and oral antidiabetic agents. Wien Klin Wochenschr. 2021;133(5-6):167-72. https://doi.org/10.1007/ s00508-019-01573-0.
14. Ishii H, Nakajima $H$, Kamei N, et al. Quality-of-life comparison of dapagliflozin versus dipeptidyl peptidase 4 inhibitors in patients with type 2 diabetes mellitus: a randomized controlled trial (J-BOND Study). Diabetes Ther. 2020;11:2959-77.

15. Suzuki M, Honda K, Fukazawa M, et al. Tofogliflozin, a potent and highly specific sodium/glucose cotransporter 2 inhibitor, improves glycemic control in diabetic rats and mice. J Pharmacol Exp Ther. 2012;341:692-701.

16. Kaku K, Watada H, Iwamoto Y, et al. The Tofogliflozin 003 Study Group. Efficacy and safety of monotherapy with the novel sodium/glucose cotransporter-2 inhibitor tofogliflozin in Japanese patients with type 2 diabetes mellitus: a combined Phase 2 and 3 randomized, placebo-controlled, double-blind, parallel-group comparative study. Cardiovasc Diabetol. 2014;13:65.

17. Tanizawa $\mathrm{Y}, \mathrm{Kaku} \mathrm{K}$, Araki E, et al; the Tofogliflozin 004 and 005 Study group. Long-term safety and efficacy of tofogliflozin, a selective inhibitor of sodium-glucose cotransporter 2, as monotherapy or in combination with other oral antidiabetic agents in Japanese patients with type 2 diabetes mellitus: multicenter, open-label, randomized controlled trials. Expert Opin Pharmacother. 2014;15:749-66.

18. Takeishi S, Tsuboi H, Takekoshi S. Comparison of tofogliflozin $20 \mathrm{mg}$ and ipragliflozin $50 \mathrm{mg}$ used together with insulin glargine $300 \mathrm{U} / \mathrm{mL}$ using continuous glucose monitoring (CGM): a randomized crossover study. Endocr J. 2017;64:995-1005.

19. Katakami N, Mita T, Yoshii H, et al. Effect of tofogliflozin on arterial stiffness in patients with type 2 diabetes: prespecified sub-analysis of the prospective, randomized, open-label, parallel-group comparative UTOPIA trial. Cardiovasc Diabetol. 2021;20(1):4. https://doi.org/10.1186/s12933-02001206-1.

20. Ishii H. Development and psychometric validation of the diabetes therapy-related QOL (DTR-QOL) questionnaire. J Med Econ. 2012;15:556-63.

21. Katakami N, Mita T, Yoshii H, et al; the UTOPIA study investigators. Rationale, design, and baseline characteristics of the UTOPIA trial for preventing diabetic atherosclerosis using an SGLT2 inhibitor: a prospective, randomized, open-label, parallel-group comparative study. Diabetes Ther. 2017;8: 999-1013. https://doi.org/10.1007/s13300-0170292-1.

22. Katakami N, Mita T, Yoshii H, et al; the UTOPIA study investigators. Tofogliflozin does not delay progression of carotid atherosclerosis in patients with type 2 diabetes: a prospective, randomized, open-label, parallel-group comparative study. 
Cardiovasc Diabetol. 2020;19:110. https://doi.org/ 10.1186/s12933-020-01079-4.

23. Editorial Committee Members of "Treatment Guide for Diabetes" (Araki E, Inagaki N, Inoguchi T, et al.). The Japan Diabetes Society: Treatment Guide for Diabetes 2014-2015. Tokyo: Bunkodo Co. Ltd.; 2014.

24. Mita T, Katakami N, Shiraiwa T, et al. The influence of sitagliptin on treatment-related quality of life in patients with type 2 diabetes mellitus receiving insulin treatment: a prespecified sub-analysis. Diabetes Ther. 2017;3:693-704.

25. Matsuo S, Imai E, Horio M, et al. Revised equations for estimated GFR from serum creatinine in Japan. Am J Kidney Dis. 2009;53:982-92.

26. Testa MA, Simonson DC. Health economic benefits and quality of life during improved glycemic control in patients with type 2 diabetes mellitus: a randomized, controlled, double-blind trial. JAMA. 1998;280:1490-6.

27. Pyatak EA, Carandang K, Vigen CLP, et al. Occupational therapy intervention improves glycemic control and quality of life among young adults with diabetes: the resilient, empowered, active living with diabetes (REAL Diabetes) randomized controlled trial. Diabetes Care. 2018;41:696-704.

28. Grandy S, Hashemi M, Langkilde AM, Parikh S, Sjostrom CD. Changes in weight loss-related quality of life among type 2 diabetes mellitus patients treated with dapagliflozin. Diabetes Obes Metab. 2014;16:645-50.

29. Grandy S, Fox KM, Bazata DD. Association of selfreported weight change and quality of life, and exercise and weight management behaviors among adults with type 2 diabetes mellitus: the SHIELD study. Cardiol Res Pract. 2012;2012: 892564. https://doi.org/10.1155/2012/892564.

30. Sarwer DB, Moore RH, Diewald LK, et al; POWERUP Research Group. The impact of a primary carebased weight loss intervention on the quality of life. Int J Obes. 2013;37:S25-S30.

31. Lean ME, Leslie WS, Barnes AC, et al. Primary careled weight management for remission of type 2 diabetes (DiRECT): an open-label, cluster-randomised trial. Lancet. 2018;391(10120):541-51.

32. Riesco E, Rossel N, Rusques C, et al. Impact of weight reduction on eating behaviors and quality of life: influence of the obesity degree. Obes Facts. 2009;2:87-95.

33. Anderson RT, Girman CJ, Pawaskar MD, et al. Diabetes medication satisfaction tool: a focus on treatment regimens. Diabetes Care. 2009;32:51-3.

34. Kleefstra N, Ubink-Veltmaat LJ, Houweling ST, Groenier KH, Meyboom-de Jong B, Bilo HJ. Crosssectional relationship between glycaemic control, hyperglycaemic symptoms and quality of life in type 2 diabetes (ZODIAC-2). Neth J Med. 2005;63: 215-21. 